\title{
Prognosis and Survival of Nasopharyngeal Cancer in Cameroon
}

\author{
Jean Paul Engbang1,2, Amadou Njifou1, Therèse Daphnée Tjomb¹, Maurice Mpessa², \\ Louis Richard Njock ${ }^{1}$
}

\author{
${ }^{1}$ Faculty of Medicine and Pharmaceutical Sciences, The University of Douala, Douala, Cameroon \\ ${ }^{2}$ Douala Laquintinie hospital, Douala, Cameroon \\ Email: jean_pen@yahoo.ca,amanjifou@yahoo.fr, laeticia.tjomb@gmail.com,mekobo@yahoo.fr,rnjock@yahoo.fr
}

How to cite this paper: Engbang, J.P., Njifou, A., Tjomb, T.D., Mpessa, M. and Njock, L.R. (2021) Prognosis and Survival of Nasopharyngeal Cancer in Cameroon. International Journal of Otolaryngology and Head \& Neck Surgery, 10, 6-19. https://doi.org/10.4236/ijohns.2021.101002

Received: November 20, 2020

Accepted: January 9, 2021

Published: January 12, 2021

Copyright $\odot 2021$ by author(s) and Scientific Research Publishing Inc. This work is licensed under the Creative Commons Attribution International License (CC BY 4.0).

http://creativecommons.org/licenses/by/4.0/

\begin{abstract}
Background: Nasopharyngeal carcinoma (NPC) is an entity belonging to upper aerodigestive tract Cancers. NPC is more widespread in Southern China and South East Asia. In our country, it is the leading cause of head and neck cancers. Its prognosis remains bleak because of the late stage at diagnostic. Objectives: The objectives of this study were to determine the prognostic factors and survival rate of patients with nasopharyngeal cancer in six reference hospitals in Cameroon. Material and Methods: It was a retrospective analytic study, conducted from January 2009 to December 2018. It was conducted in oncology, surgery and Ear Nose and Throat (ENT) units of six reference hospitals. Data from 114 files meeting the inclusion were collected. We have drawn survival curves and determined the different survival probabilities with the help of Kaplan-Meier Method. The different survival curves were compared using the Log-Rank Test $(\mathrm{P}<0.05)$, variables that were statistically associated with the $5 \%$ cut off were introduced into the Cox regression model for multivariate analysis, thus allowing us to bring out the prognostic factors significantly associated with survival. Results: The mean age at the time of diagnosis of the 114 patients recruited was $45.30 \pm 17.14$ years. The predominant histological type was the UCNT (Undifferentiated Carcinoma of the NasoPharynx) representing $84.2 \%$. According to the WHO classification, 2 patients were classified as stage I (1.8\%), 33 as stage II (28.9\%), 42 as stage III (36.8\%), 25 as stage IV A (21.9\%) and 12 as stage IV B (10.5\%). At the end of the survival assessment period, 34 patients were dead and 73 patients (64\%) were still alive. The median overall survival was 44 months. The overall survival rates at one, two, three, and four years were $80 \% ; 74 \%$; $68 \%$; $44 \%$, respectively. The prognostic factors associated with poor survival were: late consultation time of more than 12 months, N3 lymph node involvement, 3 and 4 advanced clinical stages. Conclusion: The study showed a low survival,
\end{abstract}


with a median overall survival of 44 months. The overall survival rates at one, two, three, and four years were $80 \% ; 74 \%$; $68 \%$; $44 \%$ respectively. The prognostic factors associated with poor survival were late consultation time of more than 12 months, N3 lymph Node involvement, 3 and 4 advanced clinical stages. In order to improve this survival, it is recommended that special emphasis be placed on early detection.

\section{Keywords}

Nasopharyngeal Cancer, Survival, Prognostic Factors, Detection, Cameroon

\section{Introduction}

Nasopharyngeal cancer (CNP) is a fairly rare entity $(<1 \%)$ of cancers of the upper aero digestive tract which, for their part, are ranked fifth among cancers in humans, behind prostate cancer, lung cancer, colorectal cancers and kidney and bladder cancers [1]. The very contrasting geographical distribution of this type of cancer is one of the characteristics of the disease. Globally, there are three zones: A very high frequency zone with South China (Guangzhou), where the incidence is 30 to $80 / 100,000 /$ year, and that of the north, where the incidence is 2 to 3/100,000/year; an intermediate frequency zone (8 to 12/100,000/year) with Taiwan (China), Vietnam, Thailand, Malaysia, The Philippines, The Caribbean, The Mediterranean Basin (Maghreb and Middle East), Alaska and the Greenland; And finally a low frequency zone found in Europe and the United States (0.5 to $2 / 100,000 /$ year) [2]. The peak is between 40 - 50 years old, with an often bimodal distribution ( 20 - 30 years and after 50 years) with men more frequently affected than women (sex ratio: 2.5 - 3) [3]. In 2018, 130,000 new cases of nasopharyngeal cancer were diagnosed worldwide with 73,000 reported deaths, or $1 \%$ of all cancers [4]. It presents in different histological types, dominated by carcinomas which represent more than $90 \%$ of nasopharyngeal cancers [5]. The other types, namely lymphomas of variable grade, adenoid cystic carcinomas and soft tissue tumors such as rhabdomyosarcoma, melanoma or adenocarcinoma are rarer [6]. Clinically, the manifestations of CNP are highly variable, the main warning signs being epistaxis, anosmia, nasal obstruction, serum-mucous otitis media or prevalent lymphadenopathy [7]. Given the good accessibility of the nasopharyngeal mucous space, endoscopy is the first-line exploration as it allows a positive diagnosis by performing biopsies. Magnetic Resonance Imaging (MRI) and CT scan participate in the assessment of the extension of these lesions [8] [9]. Treatment is based on radiotherapy and chemotherapy. Radiotherapy alone, which was the first curative treatment for nasopharyngeal carcinoma, remains the standard treatment for initial stage I, without lymph node involvement, with a 10 -year survival rate of $98 \%$ [10]. Regarding the prognosis, several clinical, biological and radiological factors affecting local control and survival have been 
studied; among these we note: age and sex, type and stage of tumor, EBV serology. The dose of radiotherapy and the treatment schedule have been studied primarily as a local disease control factor [11]. The prognosis of CNP remains relatively unfavorable (survival rate of around $50 \%$ at 5 years) apart from cancers classified T1 - T2 N0, due to local recurrences and metastatic relapses [12].

Concerning Cameroon, although epidemiological work carried out by Yeme $\mathrm{N}$ in 1986 showed that it is located in a low-risk zone, CNP ranks first among cancers of the ENT sphere [13] [14]. In fact, 720 cases of caval cancer had been counted over the past five years, according to GLOBOCAN 2018 data. Very little research has been carried out on this subject to our knowledge. The case of a study by Mouelle et al., on the evolutionary aspect of patients with caval cancer in 1996, found a mediocre overall survival rate of $56 \%$ at 1 year and $29 \%$, at 5 years, and a recurrence-free survival rate of $44 \%$ at 1 year [15]. Also, faced with the scarcity of local data on this condition and the frequency of which is becoming worrying in Cameroon, we see a need to contribute to the knowledge of this pathological entity, particularly in terms of prognostic factors associated with survival.

\section{Patients and Methods}

This was a retrospective analytical study, conducted from January 2009 to December 2018, in the ENT and oncology departments of six first and second category hospitals of the Cameroon health pyramid (Douala General Hospital, Douala Laquintine Hospital; Yaounde General hospital, Yaounde Central Hospital, Yaounde University Health Center, Yaounde National Social Insurance Fund Hospital Center). Histologically proven nasopharyngeal cancer patient records were included and followed during this period in these structures. Patients with other large nasopharyngeal conditions and histopathological confirmed non-malignant tumours were excluded. The various socio-demographic, clinical, paraclinical, therapeutic and evolutionary data were taken from the registers of the oncology, surgery and Ear Nose and Throat (ENT) departments of these hospitals. Patients were contacted during their follow-up visits to the hospital and those who could not report for review in the hospital were contacted via telephone. Deaths of subjects were confirmed via contact with their families and relatives. These variables were recorded and processed using SPPS version 20 software. The different associations between the variables were studied using the $\chi 2$ test or Fisher's exact test. We plotted the survival curves and determined the probabilities of survival using the Kaplan-Meier method. The comparison of the different survival curves was made using the Log-Rank test $(\mathrm{p}<0.05)$. Variables that were statistically associated with the $5 \%$ cut-off were introduced into the Cox regression model for multivariate analysis, allowing us to identify prognostic factors associated with survival.

This work had received an ethical clearance from the Ethics Committee of the University of Douala, who granted us ethical clearance No 2116 CEI-Udo/01/ 
$2020 / T$, to conduct our study in strict compliance with the ethics.

\section{Results}

\subsection{General Characteristics of the Study Population}

\subsubsection{Epidemiological Characteristics and Medical History}

We collected a total of 114 patients. The mean age at tumor diagnosis was 45.30 \pm 17.14 years, with extremes ranging from 12 to 82 years, for a median of 45.50 years. A male predominance at $66.7 \%$ (76 cases) was noted, for a sex ratio of 2.0 $\mathrm{M} / 1 \mathrm{~F}$. The majority of patients were married (81 cases, $71.1 \%$ ).

The mean time to consultation was 10 months \pm 7.25 , with extremes ranging from 3 weeks to 27 months, for a median of 9 months. A total of $36.8 \%$ of patients ( 42 cases) had already had an ENT infection, the nature of which was not specified. The patients in our series shared a diet based on smoking and curing in $86 \%$ of cases (98 patients). The family history of nasopharyngeal cancer was noted in $1.8 \%$ of cases ( 2 patients) (Table 1 ).

\subsubsection{Clinical Signs}

As is shown in Table 2, the clinical picture was dominated by the appearance of a tumor syndrome found in $82.5 \%$ of patients (94 cases). Our patients could also present in an isolated or associated way rhinological, otological or neurological signs.

Among the $92.1 \%$ (105 cases) who presented cervical lymphadenopathy, it was objectified that they were, in 48.6\% (51 cases), of a size between 3 and $6 \mathrm{~cm}$, their site was mainly jugulocarotid in $57.1 \%$ (60 cases), and in $71.4 \%$ of cases (70 patients), they were of bilateral topography.

The auditory examination carried out by otoscopy mainly revealed serum-mucous otitis in $87 \%$ of the patients ( 87 cases) in our series.

Exploration of the rhinological system was done with a nasofibroscope and revealed an ulcerative budding lesion in $80 \%$ of cases ( 80 patients).

\subsubsection{Histolopathological and Treatment Characteristics}

UCNT (Undifferentiated Carcinoma of the NasoPharynx) was the predominant histologic type in $84.2 \%$ of patients ( 80 cases), while Non-Hodgkin's Malignant Lymphomas (NHLM) were the least represented histologic type with $3.5 \%$ of patients (4 cases) (Table 3). Patients were classified according to the TNM classification adopted by the AJCC Cancer Staging Manual 8th edition (2017). We found, for clinical tumor size, a predominance of patients classified T2 and T3, respectively $49.1 \%$ and $29.8 \%$ of cases (56 cases and 34 cases). N2 lymph node invasion was predominantly observed, and $21.9 \%$ of patients ( 25 cases) developed metastases. In our series, $69.2 \%$ of patients (79 cases) were diagnosed in stages III and IV, on the other hand only $1.8 \%$ ( 2 cases) were grouped together in stage I (Table 3).

The main therapeutic modalities found were concomitant radio chemotherapy and exclusive radiotherapy administered respectively in $47.4 \%$ and $31.5 \%$ of cases (54 and 36 patients). 
Table 1. General characteristics and medical history of patients.

\begin{tabular}{|c|c|c|}
\hline Variable & $\mathbf{N}$ & $\%$ \\
\hline \multicolumn{3}{|l|}{ Age } \\
\hline $10-19$ & 16 & 13.9 \\
\hline $20-29$ & 9 & 7.9 \\
\hline $30-39$ & 15 & 13.0 \\
\hline $40-49$ & 26 & 22.6 \\
\hline $50-59$ & 23 & 20.0 \\
\hline$\geq 60$ & 25 & 21.7 \\
\hline \multicolumn{3}{|l|}{ Gender } \\
\hline Male & 76 & 66.7 \\
\hline Female & 38 & 33.3 \\
\hline \multicolumn{3}{|l|}{ Consultation delay } \\
\hline$<1$ & 9 & 7.9 \\
\hline $2-6$ & 30 & 26.3 \\
\hline $7-12$ & 30 & 26.3 \\
\hline$>12$ & 45 & 39.5 \\
\hline \multicolumn{3}{|l|}{ Comorbidities } \\
\hline ENT infection & 42 & 36.8 \\
\hline AHT & 15 & 13.2 \\
\hline Diabetes & 9 & 7.9 \\
\hline HIV & 3 & 2.6 \\
\hline Epigastralgia & 2 & 1.8 \\
\hline Hepatitis B & 2 & 0.9 \\
\hline Chronic sinusitis & 1 & 0.9 \\
\hline Tuberculosis & 1 & 0.9 \\
\hline \multicolumn{3}{|l|}{ Surgical history } \\
\hline Cervical adenectomy & 1 & 0.9 \\
\hline \multicolumn{3}{|l|}{ Toxicological history } \\
\hline Alcoholic intake & 15 & 13.2 \\
\hline Smoking history & 8 & 7.0 \\
\hline \multicolumn{3}{|l|}{ Diet } \\
\hline Smoked fish/meat & 98 & 86.0 \\
\hline Spicy food & 71 & 62.3 \\
\hline Very salty meat fish & 1 & 0.9 \\
\hline \multicolumn{3}{|l|}{ Family history } \\
\hline Cavum cancer & 2 & 1.8 \\
\hline Breast cancer & 1 & 0.9 \\
\hline Cervical cancer & 1 & 0.9 \\
\hline Thyroid cancer & 1 & 0.9 \\
\hline
\end{tabular}


Table 2. Symptoms and physical signs of the study population.

\begin{tabular}{ccc}
\hline Symptoms/Signs & N & $\%$ \\
\hline Cervical swelling & 94 & 82.5 \\
Rhinological Syndrome & & \\
Nasal obstruction & 53 & 46.5 \\
Epistaxis & 46 & 40.4 \\
Otologic syndrome & & \\
Hearing loss & 50 & 43.9 \\
Tinnitus & 19 & 16.7 \\
Otalgia & 18 & 15.8 \\
Neurological syndrome & & \\
Headache & 50 & 34.8 \\
Facial neuralgia & 4 & 3.5 \\
Trismus & 2 & 1.6 \\
Cervical lymphadenopathy & 51 & 48.6 \\
Nasofibroscopy & & \\
Budding ulcerative lesion & 13 & 84.2 \\
Infiltrating & 2 & 2.1 \\
Polypoid & &
\end{tabular}

Table 3. Histopathological and treatment characteristics.

\begin{tabular}{ccc}
\hline Variable & N & $\%$ \\
\hline Histological types & & \\
WHO type III (UCNT) & 80 & 84.2 \\
WHO type I & 12 & 10.5 \\
WHO type II & 9 & 7.9 \\
LMNH & 4 & 3.5 \\
\hline Stages & & \\
I & 2 & 1.8 \\
II & 33 & 28.9 \\
III & 42 & 36.8 \\
IVA & 25 & 21.9 \\
IVB & 12 & 10.5 \\
\hline Treatment & & \\
\hline 1st chemotherapy + Locking radiotherapy & 54 & 47.4 \\
Ealliative chemotherapy & 36 & 31.5 \\
\hline Concomitant radiochemotherapy & 13 & 11.4 \\
\hline
\end{tabular}




\subsection{Survival}

As shown in Figure 1, the median overall survival was 44 months with a confidence interval ( $95 \% \mathrm{CI})$ of (37.56 - 50.43). The overall survival rates at one, two, three and four years were $80 \%$, respectively; $74 \%$; $68 \%$; $44 \%$.

\subsection{Pronostic Factors}

\subsubsection{Univariate Analysis}

In univariate analysis, some factors were found to be significant; in particular, young age (10 - 20 years), and advanced age (over 60 years), consultation time of less than one month and those over 7 and 12 months, locally advanced tumor size (T3 and T4), lymph node invasion (N2 and N3), presence of metastases, WHO stages 3 and 4 , palliative chemotherapy, conformational radiotherapy, dosimetry $\geq 65 \mathrm{~Gy}$, spreading over 6 weeks of radiotherapy (Table 4).

\subsubsection{Multivariate Analysis}

After multivariate analysis by calculating the Hazard Ratio of each factor using the Cox model, it emerges that the factors associated with death were: The late consultation period of more than 12 months, N3 lymph node invasion, Stages 3 and 4 cancer (Table 5).

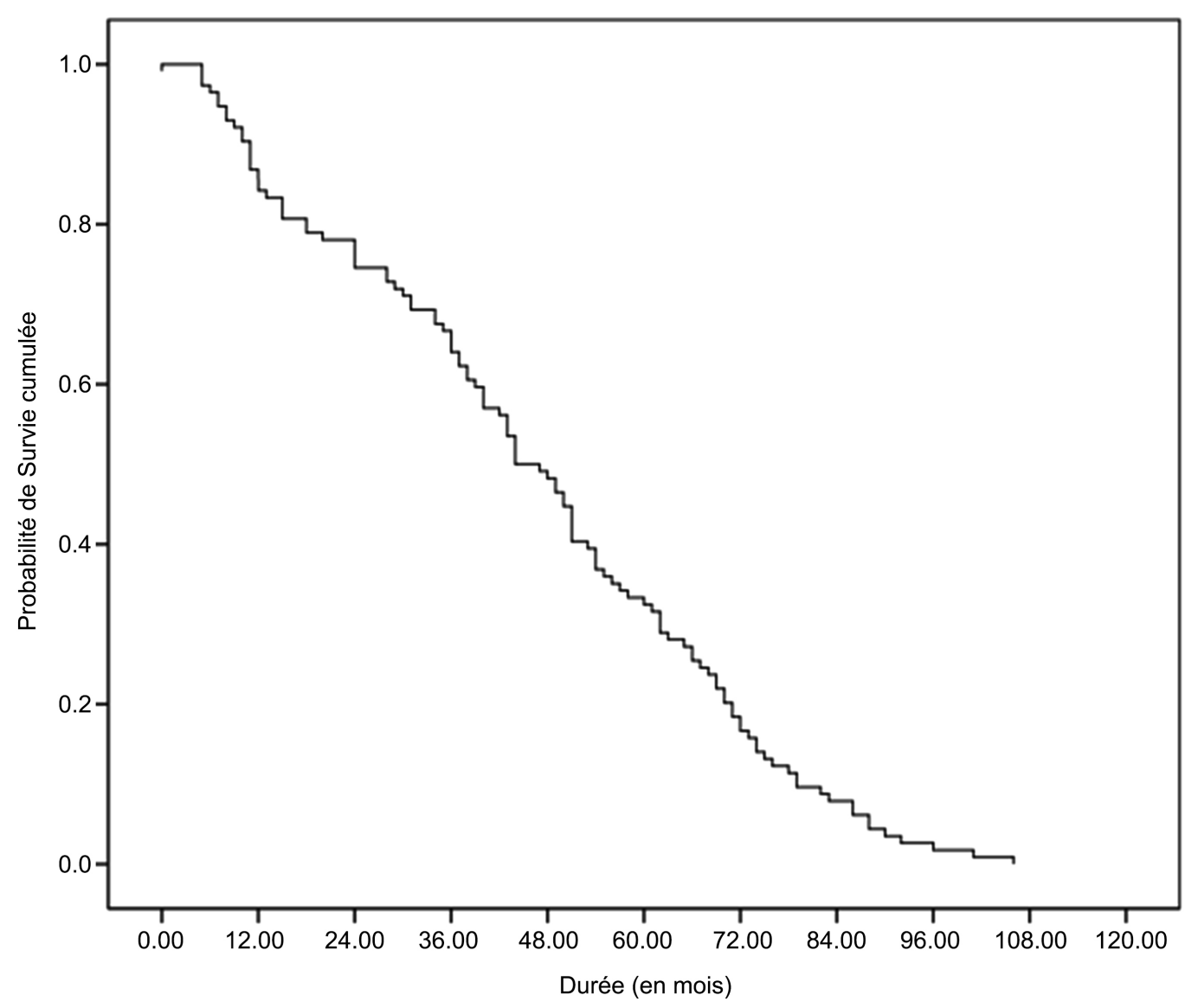

Figure 1. Overall survival curve of the study population. 
Table 4. Univariate analysis of prognostic factors.

\begin{tabular}{|c|c|c|}
\hline Variable & Median OS (95\% CI) (months) & p-value \\
\hline \multicolumn{3}{|l|}{ Age (years) } \\
\hline$\geq 60$ & $18(5.47-30.52)$ & $<0.0001^{*}$ \\
\hline \multicolumn{3}{|l|}{ Consultation delay (months) } \\
\hline $7-12$ & $49(42.73-55.26)$ & $0.032^{\star}$ \\
\hline$>12$ & $30(20.79-39.20)$ & $<0.0001^{*}$ \\
\hline \multicolumn{3}{|l|}{ Tumor size } \\
\hline $\mathrm{T} 3$ & $35(50.00-73.99)$ & $0.006^{*}$ \\
\hline $\mathrm{T} 4$ & $18(9.39-26.60)$ & $<0.0001^{*}$ \\
\hline \multicolumn{3}{|l|}{ Lymph node metastasis $(\mathrm{N})$} \\
\hline $\mathrm{N} 2$ & $50(47.31-52.68)$ & $<0.0001^{*}$ \\
\hline $\mathrm{N} 3$ & $24(14.65-33.93)$ & $<0.0001^{*}$ \\
\hline \multicolumn{3}{|l|}{ Distant Metastasis (M) } \\
\hline yes & $30(17.12-42.85)$ & $0.041^{*}$ \\
\hline \multicolumn{3}{|l|}{ Stage } \\
\hline III & $50(47.24-52.75)$ & $0.002^{*}$ \\
\hline IV & $20(10.70-29.29)$ & $<0.0001^{\star}$ \\
\hline \multicolumn{3}{|l|}{ Treatment } \\
\hline Palliative chemotherapy & $24(15.31-32.68)$ & $<0.001^{\star}$ \\
\hline \multicolumn{3}{|l|}{ Type of radiotherapy } \\
\hline Conformational & $44(38.13-49.86)$ & $<0.001^{*}$ \\
\hline \multicolumn{3}{|l|}{ Radiation therapy dose } \\
\hline$\geq 65 \mathrm{~Gy}$ & $51(45.05-56.94)$ & $<0.001^{\star}$ \\
\hline \multicolumn{3}{|l|}{ Staggered radiotherapy } \\
\hline$\geq 6$ weeks & $51(45.42-56.57)$ & $<0.001^{\star}$ \\
\hline \multicolumn{3}{|l|}{ Chemotherapy protocol } \\
\hline CDDP + Doxoribucin & $88(15.0-161)$ & $0.027^{\star}$ \\
\hline
\end{tabular}

Table 5. Multivariate analysis.

\begin{tabular}{ccc}
\hline Variable & HR (IC 95\%) & p-value \\
\hline Consultation delay (months) & $10.03(1.64-21.18)$ & $0.012^{\star}$ \\
Lymph node involvement (N) & & \\
N3 & $3.83(0.88-16.62)$ & $0.018^{\star}$ \\
Stage & & \\
IV & $24.38(7.72-76.99)$ & $<0.001^{*}$ \\
\hline
\end{tabular}




\section{Discussion}

In our study, the overall survival rates at one, two, three, and four years were $80 \%, 74 \%$; $68 \%$; $44 \%$ respectively. Our results at one year are similar to those found by Soundouss Raissouni in 2009 in Morocco, Kwong et al. in Hong Kong in 2006, Tham et al. in Singapourg in 2009, which had respectively values of $81 \%, 86.5 \%$ and $88 \%$ [16] [17] [18]. Our overall 4 -year survival rate of $44 \%$ is comparable to those found by Soundouss Raissouni in 2009 in Morocco, Haddaoui et al. in Tunisia in 2013, which had respective values of 38\% [16] and 50\% [19]. However, it was lower than those found by Sultanem et al. In the United States in 2000; Kwong et al. In Hong Kong in 2006; Tao et al. in China in 2013, with their respective values of $82 \%, 78 \%, 80.6 \%$ [17] [20] [21]. This difference could be explained by the fact that, in our context, the diagnosis of nasopaharyngeal cancer are more often made at an advanced stage (III and IV in $69.2 \%$ of our cases), thus compromising the patient's prognosis. Apart from the anatomical reasons mentioned above in our context, this late diagnosis can be explain, by the negative effect of the ignorance and the poverty of the patients, by their sometimes impossibility of having access to specialized hospitals which are concentrated in the main cities, by the insufficient number of doctors and cancer specialists and by diagnostic errors following numerous consultations with traditional practitioners, houses of prayer and self-medication. Moreover, it was better than those found by Yomi et al. in Cameroon in 1995; Mouelle et al. in Cameroon in 1996; Hind C in Senegal in 2013; Mamadou G in Senegal in 2017, who found respective values of $34 \%, 36 \%, 0 \%$ and $26 \%$ [15] [22] [23] [24]. This could be explained by the fact that, despite the late diagnosis in our context, progress has been made in recent years in the management of caval cancer, in particular by the adoption of new therapeutic strategies in terms of radiotherapy (use of higher doses with more suitable irradiation of the cervical lymph node areas) but also chemotherapy (neoadjuvant chemotherapy and new protocols).

After running the Cox regression model, and calculating the adjusted Hazard Ratio of death for each factor identified by univariate analysis and comparison of survival curves using the Log-Rank test, the relevant prognostic factors that remained associated with the survival with bad prognosis were the late consultation period of more than 12 months, N3 lymph node invasion and stages 3 and 4 of cancer. Our results are comparable to those of Haldum et al. in Turkey in 2001, they showed in a series of 357 patients with caval cancer including 272 of WHO type 3 that lymph node involvement was a major prognostic factor in terms of disease-free survival and overall survival [25]. Chua et al. have also shown in a study including 290 patients in China in 1997 where they calculated lymph node volume as an important factor affecting remote control and disease-free survival [26]. Mu-Tai Liu in China in 2003 had also shown that lymph node status was an important prognostic factor for overall survival [27]. Likewise, Earnest A. et al. in a meta-analysis in 2012 in Singapore demonstrate that lymph node involvement is by far the determining prognostic factor for survival, 
in both adults and children [28].

Chen Cheng et al. in China found that stage was an important prognostic factor in terms of relapse-free survival [29].

In Jouin Bortolotti's series in 2016 in France, the delay in diagnosis appears to be the only risk factor affecting overall survival [30]. In fact, in our series, $65.8 \%$ of cases (75 patients) consulted after a period of 7 months, which explains the diagnostic delay and the advanced stages of our series. In addition, we found in our series that patients without lymphatic involvement, or whose involvement was limited to stage 1 , had better survival than those with lymph node invasion stage 3. Moreover, Patients diagnosed with WHO stage 1 had better survival, compared to those diagnosed at stages 3 and 4 .

In our study, age did not appear as a prognostic factor affecting overall survival; which is similar to the findings of Soundouss Raissinou in Morocco in 2009 and Mamadou G in Senegal in 2017. On the other hand, in the study of Haldun et al. it was established that adolescents and young adults had a better prognosis than the elderly [25]. This difference could be explained by the fact that the age group under 20 represents only $13.9 \%$ of our study population.

Concomitant radiochemotherapy was not a prognostic factor associated with survival in our study; unlike those of Yeh et al.; Zeng et al., who showed that local control of the tumor was dependent on the action of radiotherapy combined with chemotherapy [31] [32]. We did not find any benefit in doing concomitant radiochemotherapy over radiotherapy in our series. This is probably due to the bias of the retrospective study and to the unbalance of the two treatment arms: the number of patients and the characteristics of the patients (given that 51.8\% of the patients in the concomitant radiochemotherapy group were of III and IVA). This unperceived benefit can also be explained by the fact that the decision to combine radiotherapy with chemotherapy is taken late.

The dose of radiotherapy to the cavum has been studied extensively, especially as a local controlling factor. This factor would be all the more important as the tumor is more developed locally. Bedwineck et al. did not report a change in the local control rate of tumors classified $\mathrm{T} 1$ and $\mathrm{T} 2$ if the irradiation dose delivered was greater than $60 \mathrm{~Gy}$. However, they showed that, for tumors classified T3 and $\mathrm{T} 4$, there was a linear increase in local control from 55 Gy to 75 Gy [33].

Fang et al. observed in their study an increase in local control rates at three years for tumors classified as T3 and T4, with an increase in the dose delivered. For these, the rates were $46 \%$ and $63 \%$, respectively, with irradiation doses less than and greater than $75.6 \mathrm{~Gy}(\mathrm{p}=0.02)$ [29]. For Lee et al. the risk of relapse was 1.16 for patients who received an irradiation dose between 60 and $63 \mathrm{~Gy}$ and 1.86 for those who received a dose between 55 and $59 \mathrm{~Gy}$, compared to patients who had a dose greater than $64 \mathrm{~Gy}(\mathrm{p}=0.008)$ [34] [35]. For the same authors, the risk of local relapse decreased by $9 \%$ per additional Gy in the tumor from a dose of $45 \mathrm{~Gy}$. In our series, the radiation dose was not a prognostic factor affecting the overall survival of patients. 


\section{Limitations of the Study}

The main limitation of our study comes from its retrospective nature and the biases associated with this type of methodology. Indeed, we encountered difficulties in the use of files linked to insufficient data, in particular with regard to biological data such as EBV serology, which is a major prognostic factor because it is associated with nasopharyngeal cancer in Literature. Or radiological data such as tumor volume obtained from CT images by calculating the sum of the different diameters, which constitutes a prognostic factor affecting local control of the disease. The second difficulty was linked to a follow-up bias due to the fact that there are only two radiotherapy centers in Cameroon: The Douala General Hospital and the Bekoko center were not included in our sites. As a result of the closed up of the Yaounde radiotherapy center since 2012, patients diagnosed in a structure other than the Douala General Hospital were systematically referred to one of the two centers for further treatment, giving a problem of file traceability. This significantly affected our data collection. Despite these limitations, this study made it possible to assess the prognostic factors of cavum cancer in six referral hospitals in the cities of Douala and Yaoundé.

\section{Conclusion}

Nasopaharyngeal cancer (NPC) is diagnosed at a relatively young age. Survival was poor, with a median overall survival of 44 months; the overall survival rates at one, two, three, four years were $80 \%$, respectively; $74 \% ; 68 \% ; 44 \%$. Poor prognosis factors identified were time of consultation of more than 12 months, N3 lymph node invasion, stages 3 and 4 of cancer. In order to improve survival, it is recommended to place particular emphasis on early detection as well as the use of new therapies.

\section{Conflicts of Interest}

The authors declare no conflicts of interest regarding the publication of this paper.

\section{References}

[1] Lefebvre, J. and Chevalier, D. (2012) Épidémiologie des cancers des voies aérodigestives supérieures. EMC Oto-rhino-laryngologie, 7, 1-11. https://doi.org/10.1016/S0246-0351(12)41900-6

[2] Chang, E. and Adami, H. (2006) The Enigmatic Epidemiology of Nasopharyngeal Carcinoma. Cancer Epidemiology, Biomarkers \& Prevention, 15, 1765-1777. https://doi.org/10.1158/1055-9965.EPI-06-0353

[3] Yu, M. and Yuan, J. (2006) Epidemiology of Nasopharyngeal Carcinoma. Seminars in Cancer Biology, 12, 421-429. https://doi.org/10.1016/S1044579X02000858

[4] Ferlay, J., Bray, F., et al. (2018) Cancer Incidence and Mortality Worldwide: Sources, Methods and Major Patterns in GLOBOCAN. International Journal of Cancer, 136, 359-386. https://doi.org/10.1002/ijc.29210

[5] Wei, W. and Sham, J. (2005) Nasopharyngeal Carcinoma. The Lancet, 365, 2041-2054. 
https://doi.org/10.1016/S0140-6736(05)66698-6

[6] Boussen, H., Bouaouina, N., Gamoudi, A., Mokni, N., Benna, F., Boussen, I., et al. (2007) Cancers du nasopharynx. EMC (Elsevier Masson SAS, Paris), Oto-rhino-laryngologie, 20-590-A-10, $23 \mathrm{p}$. https://doi.org/10.1016/S0246-0351(07)41921-3

[7] Rivera, A., Keryer, P. and Busson, C. (2005) Les carcinomes du nasopharynx: De la biologie à la clinique. Cancerl Radiothérapie, 182, 55-68. https://doi.org/10.1016/j.canrad.2004.12.005

[8] Chong, V., Fan, Y. and Khoo, J. (1996) Nasopharyngeal Carcinoma with Intracranial Spread: CT and MR Characteristics. Journal of Computer Assisted Tomography, 20, 563-569. https://doi.org/10.1097/00004728-199607000-00012

[9] Chung, N., Ting, L., Hsu, W., Lui, L. and Wang, P. (2004) Impact of Magnetic Resonance Imaging versus CT on Nasopharyngeal Carcinoma: Primary Tumor Target Delineation for Radiotherapy. Head \& Neck, 26, 241-246.

https://doi.org/10.1002/hed.10378

[10] Chua, D., Sham, J. and Kwong, D. (2003) Treatment Outcome after Radiotherapy Alone for Patients with Stage I-II Nasopharyngeal Carcinoma. Cancer, 98, 74-80. https://doi.org/10.1002/cncr.11485

[11] Kim, S., Smith, B. and Haffty, B. (2014) Prognostic Factors in Patients with Head and Neck Cancer. In: Harrison, L.B., Sessions, R.B. and Kies, M.S., Eds., Head and Neck Cancer. A Multidisciplinary Approach, Wolters Kluwer, Philadelphia, 87-111.

[12] Frikha, M., Bouaziz, M., Daoud, J., Ghorbel, A., Ellouni, M., Boudaya, M., et al. (1997) Evaluation de la réponse tumorale et ganglionnaire à la chimiothérapie néoadjuvante dans les carcinomes indifférenciés du nasopharynx. Bulletin du Cancer, 84, 273-276.

[13] Njifou, A., Njock, L., Ngnembi, A., Essama, L., Fewou, A., Minka, E., et al. (2018) Aspects Anatomopathologiques des Cancers ORL et Cervico-faciaux à l'Hôpital Général de Douala. Health Sciences, 19, 44-45.

[14] Yeme, N. (1986) Le cancer du cavum à Yaoundé: Prevalence, attitude clinique et approche etiopathogenique. Thèse de médecine, Cuss, Yaounde (Cameroun).

[15] Mouelle, A., Essomba, M., Fouda, A. and Ndom, P. (2001) Aspects évolutifs des cancers du Cavum traités à l'hôpital général de Douala. Médecine d Afrique Noire, 48, 98-101.

[16] Yomi, J., Fouda, A., Maloum, E. and Bengono, G. (1995) Carcinomes du cavum à Yaoundé. Bulletin du Cancer/ Radioth, 82, 396-398. https://doi.org/10.1016/0924-4212(96)80056-9

[17] Hind, C. (2013) Cancer du nasopharynx (CAVUM). A propos de 102 cas colligés à l'institut Joliot Curie de Dakar. Thèse de médecine, Dakar (Sénégal), No. 54, 93 p.

[18] Mamadou dit Doudou Gueye (2017) Carcinomes du cavum à l'institut Joliot Curie de Dakar: Etude retrospective de 22 cas (Janvier 2009-Décembre 2015). Thèse de médecine, Dakar, No. 235, 108 p.

[19] Haddaoui, A., Ayari, J. and Balti, M. (2013) Carcinomes nasopharyngés localement avancés. Journal Tunisien d ORL et de Chirurgie Cervico-Faciale, No. 30, 47-52.

[20] Soundouss, R. (2009) Facteurs pronostiques du carcinome du cavum localement avancé. Thèse de médecine, Rabat: Maroc, No. 109, 96 p.

[21] Kwong, D., Sham, J., Leung, L., Cheng, A., Ng, W., Kwong, P., et al. (2006) Preliminary Results of Radiation Dose Escalation for Locally Advanced Nasopharyngeal Carcinoma. International Journal of Radiation Oncology Biology Physics, 64, 374- 
381. https://doi.org/10.1016/j.ijrobp.2005.07.968

[22] Tham, W., Hee, S., Yeo, R., Salleh, P., Lee, J., Tan, T., et al. (2009) Treatment of Nasopharyngeal Carcinoma Using Intensity-Modulated Radiotherapy-The National Cancer Centre Singapore Experience. International Journal of Radiation Oncology Biology Physics, 75, 1481-1486.

https://doi.org/10.1016/j.ijrobp.2009.01.018

[23] Sultanem, K., Shu, H., Xia, P., Akazawa, C., Quivey, J., Verhey, L., et al. (2000) ThreeDimensional Intensity-Modulated Radiotherapy in the Treatment of Nasopharyngeal Carcinoma: The University of California-San Francisco Experience. International Journal of Radiation Oncology Biology Physics, 48, 711-722. https://doi.org/10.1016/S0360-3016(00)00702-1

[24] Tao, C., Liu, X., Tang, L., Mao, Y., Chen, L., Li, W., et al. (2013) Long-Term Outcome and Late Toxicities of Simultaneous Integrated Boost-Intensity Modulated Radiotherapy in Pediatric and Adolescent Nasopharyngeal Carcinoma. Chinese Journal of Cancer, 32, 525-532. https://doi.org/10.5732/cjc.013.10124

[25] Haldun, S. and Erkal, J. (2001) Nasopharyngeal Carcinomas: Analysis of Patient, Tumor and Treatment Characteristics Determining Outcome. Radiotherapy and Oncology, 61, 247-256. https://doi.org/10.1016/S0167-8140(01)00448-0

[26] Chua, D.T., et al. (1997) Volumetric Analysis of Tumor Extent in Nasopharyngeal Carcinoma and Correlation with Treatment Outcome. International Journal of Radiation Oncology, Biology, Physics, 39, 711-719. https://doi.org/10.1016/S0360-3016(97)00374-X

[27] Liu, M.T. (2003) Prognostic Factors Affecting the Outcome of Nasopharyngeal Carcinoma. Japanese Journal of Clinical Oncology, 33, 501-508. https://doi.org/10.1093/jico/hyg092

[28] Earnest, A., Ho, F. and Tham, I. (2012) Patterns of Regional Lymph Node Metastasis of Nasopharyngeal Carcinoma: A Meta-Analysis of Clinical Evidence. BMC Cancer, 12, 98. https://doi.org/10.1186/1471-2407-12-98

[29] Fang, F., Leung, S. and Chen, H. (1999) Computed Tomography Findings of Bony Regeneration after Radiotherapy for Nasopharyngeal Carcinoma with Skull Base Destruction Implications for Local Control. International Journal of Radiation Oncology, Biology, Physics, 44, 305-309. https://doi.org/10.1016/S0360-3016(99)00004-8

[30] Bortolotti, J. (2016) Etude rétrospective multicentrique évaluant l'impact de l'étalement de la radiothérapie dans les cancers indifférenciés du cavum non métastatique de l'enfant et du jeune adulte. Thèse de médecine, Université de Lille, Lille, 94 p.

[31] Yeh, S., Tang, Y. and Lui, C. (2006) Treatment Outcomes of Patients with AJCC Stage IVC Nasopharyngeal Carcinoma: Benefits of Primary Radiotherapy. Japanese Journal of Clinical Oncology, 36, 132-136. https://doi.org/10.1093/jico/hyi245

[32] Zeng, L., Tian, Y., Huang, Y., Sun, M., Wang, F., Deng, X., et al. (2014) Retrospective Analysis of 234 Nasopharyngeal Carcinoma Patients with Distant Metastasis at Initial Diagnosis: Therapeutic Approaches and Prognostic Factors. PLoS ONE, 9, e108070. https://doi.org/10.1371/journal.pone.0108070

[33] Bedwinek, J., Perez, C. and Keys, D. (1980) Analysis of Failures after Definitive Irradiation for Epidermoid Carcinoma of the Nasopharynx. Bulletin du Cancer, 45, 2725-2729.

https://doi.org/10.1002/1097-0142(19800601)45:11<2725::AID-CNCR2820451105> 3.0.CO;2-1

[34] Lee, A., Law, S. and Foo, W. (1993) Retrospective Analysis of Patients with Naso- 
pharyngeal Carcinoma Treated during 1976-1985 Survival after Local Recurrence. International Journal of Radiation Oncology, Biology, Physics, 26, 773-782. https://doi.org/10.1016/0360-3016(93)90491-D

[35] Lee, A., Law, S., Foo, W. and Poon, Y. (1993) Nasopharyngeal Carcinoma: Local Control by Megavoltage Irradiation. The British Journal of Radiology, 66, 528-536. https://doi.org/10.1259/0007-1285-66-786-528 